Al-Azhar Bull. Sci. Vol. 19, No. 2 (Dec.): pp. 213-224, 2008.

\title{
SYNTHESIS OF 4-HYDROXY-3-SUBSTITUTED COUMARINS OF EXPECTED BIOLOGICAL ACTIVITY AND THEIR REACTIONS WITH SOME NUCLEOPHILES
}

NAHED F. ABD EL-GHAFFAR, NADIA. T. DAWOOD, , FATMA,H. ABD ELSALAM AND AWTIF E.FARRAG

Chemistry Department, Faculty of Science, Al-Azhar University, Nasr City, Cairo

Abstract

3-Acetyl-4-hydroxy substituted coumarins $\left(\mathbf{I I}_{\mathbf{a}-\mathbf{c}}\right)$ were prepared. Their reactions with hydrazines in DMF gave the corresponding 4-hydroxy -(3-substituted pyrazolin-3-yl)coumarins $\left(\mathbf{I V}_{\mathbf{a}-\mathbf{f}}\right)$ via the intermediate hydrazone (III). Their reaction with hydroxylamine hydrochloride gave the corresponding isoxazol-3-yl coumarin $\left(\mathbf{V I}_{\mathbf{a}-\mathrm{c}}\right)$ and $\left(\mathbf{V I I}_{\mathbf{a}, \mathbf{b}}\right)$ via the oxime intermediate $(\mathbf{V})$. Reaction of II with aromatic aldehydes gave the corresponding chalcone derivatives $\left(\right.$ VIII $_{\mathbf{a}-\mathbf{k}}$ ) which were reacted with hydrazines and hydroxyl amine hydrochloride and gave $\left.\mathbf{I X}_{\mathbf{a}}\right)$ and $\left(\mathbf{X}_{\mathrm{a}-\mathrm{c}}\right)$. Condensation of $\left(\mathbf{I I}_{\mathrm{a}-\mathrm{c}}\right)$ with aromatic aldehydes, malononitrile in the presence ammonium acetate yielded the corresponding 3-cyano-4-aryl nictoinamido-6-ylcoumarin $\left(\mathbf{X I}_{\mathbf{a}-\mathrm{f}}\right)$.

4-Hydroxy comumarin $\left(\mathbf{1}_{\mathrm{a}-\mathrm{c}}\right)$ on treatment with $\mathrm{POCl}_{3}$ in $\mathrm{DMF}$ give the corresponding 4-Chloro-3-formyl coumarin $\left(\mathbf{X I I}_{\mathbf{a}-\mathrm{c}}\right)$ which on treatment with sod azide gave the corresponding 4-azido-3-formyl coumarin ( $\left.\mathbf{X I I I}_{\mathrm{a}-\mathrm{c}}\right)$. 4-chloro-3formyl coumarine (XII )on treatment with hydrazine gave pyrazolo coumarin derivatives $\left(\mathbf{X I V}_{\mathbf{a}-\mathbf{d}}\right)$ and on treatment with hydroxylamine hydrochloride gave 4chloro-3-aldo oxime coumarin derivatives $\left(\mathbf{X} \mathbf{V}_{\mathbf{a}, \mathbf{b}}\right)$. On treatment of 4-azido-3formyl coumarin with active methylene compounds such as malononitrile and ethyl cyanocetate derivatives gave $\left(\mathbf{X V I}_{\mathbf{a}-\mathbf{d}}\right)$ respectively.

The antibacterial and antifungal activities of some compounds have been described.

\section{Results and Discussion :}

Coumarins are versatile reagents and their utility in heterocyclic synthesis has recently received a considerable attention. They possess significant biological activities $^{(1-3)}$, anti-bacterial ${ }^{(4)}$, anti-tumoral ${ }^{(5)}$, and anti-HIv ${ }^{(6-9)}$. Due to those varied biological activities, we have synthesized several 4-substituted, 4,3-substituted-2H1-benzopyran-2-one derivatives in order to investigate the structure activity relationships of coumarin derivatives. In the present investigation, we report here the synthetic procedures for compounds (I-IV) and the anti-microbial activity of the products. Thus 4-hydroxycoumarins $\left(\mathbf{I}_{\mathbf{a}-\mathbf{c}}\right)$ under suitable procedures yielded 3- 
acetyl-4-hydroxycoumarin $\left(\mathbf{I I}_{\mathbf{a}-\mathbf{c}}\right)$, which reacted with hydrazines, namely hydrazines hydrate, phenyl hydrazine and 4-nitrophenyl hydrazine in boiling DMF containing few drops of piperidine to give the pyrazole derivative ( $\left.\mathbf{I V}_{\mathrm{a}-\mathrm{f}}\right)$. The reaction of (II) with hydroxylamine hydrochloride in boiling ethanol containing sodium acetate yielded the oximes $(\mathbf{V})$ which on reaction with $\mathrm{DMF} /$ piperidine in boiling xylene gave the corresponding isoxazol-3-yl coumrains $\left(\mathbf{V I}_{\mathbf{a}-\mathbf{c}}\right)$ and $\left(\mathbf{V I I} \mathbf{I}_{\mathbf{a}, \mathbf{b}}\right)$ respectively. It is believed that compounds $\left(\mathbf{V I}_{\mathrm{a}-\mathrm{c}}\right)$ are formed via nucleophilic attack of nitrogen of the hydroxylamine moiety the carbonyl of compounds (II) and the formyl groups of DMF affected cyclization to the desired isoxazole derivatives (VI) and may be a little of $\left(\mathbf{V I I}_{\mathbf{a}, \mathbf{b}}\right)$.

Structure (VI) was established on the basis of ${ }^{1} \mathrm{H}-\mathrm{NMR}$ spectrum as the 4hydroxy proton was observed at $\delta 12.8 \mathrm{pmm}$ and the absence of the methyl protons.

The base catalyzed reaction of (II) with aromatic aldehydes ${ }^{(10,11)}$ in chloroform in the presence of piperidine with azostropic removal of water afforded the corresponding 4-hydroxy-3-cinnamoyl coumarin derivatives $\left(\mathbf{V I I I}_{\mathrm{a}-\mathrm{k}}\right)^{(12)}$. The ${ }^{1} \mathrm{H}$ NMR spectrum of $\left(\mathbf{V I I I}_{\mathbf{a}}\right)$ showed the coupling of the olefinic protons at $\delta 5.95$ and $\delta 8.55 \mathrm{ppm}(\mathrm{J}=15 \mathrm{~Hz}, \mathrm{AB}$ system $)$. The reaction of VIII with malononitrile in refluxing ethanol containing ammonium acetate yielded the corresponding 4hydroxy-3 (3-cyano-4-aryl-2(1H)-pyridine)-yl coumarins $\left(\mathbf{X I}_{\mathbf{a}-\mathbf{f}}\right)$. The formation of XI-might proceed via an intermediate reaction between $\beta$-aminoenones and malononitrile to give 2(1H)-pyridone (XI) and considered to begin with a conjugated addition of the nitrile to the enone followed by elimination of water and cyclization to (XI). This was further proved by the reaction of (II) with aromatic aldehydes namely 4-nitrobenzaldehyde, 2,4-dimethylamino benzaldehyde, furfural and/or isonicotinaldehyde to give XI. This was in agreement with the previous findings ${ }^{(13)}$.

The reaction of 3-cinnamoyl coumarins VIII with hydrazines namely, hydrazine hydrate, phenyl hydrazine and/or 4-nitro phenyl-hydraine in boiling ethanol afforded the corresponding pyrazoline derivatives $\mathbf{I X}_{\mathbf{a}-\mathbf{h}}$; while the reaction of compounds VIII with hydrazine hydrate in refluxing glacial acetic acid, it affected acetylation of $\mathrm{NH}$ of the pyrazoline ring and gave the corresponding 4-hydroxy-3-[(N-acetyl) pyrazoline]-3-yl coumarin IXi,j. Similarly, the reaction of VIII with hydroxylamine hydrochloride in boiling pyridine gave the corresponding 3-(isoxazol)-5-yl coumarin $\left(\mathbf{X}_{\mathrm{a}-\mathrm{c}}\right)$. 
The formation of IX and $\mathbf{X}$ were assumed to proceed via initial nucleophilic attack of the amino group to the carbonyl of the cinnamoyl moiety without attack at the carbonyl of the $\alpha$-pyrone ring followed by cyclization.

The present investigation also deals with the action of halogen nucleophiles on the $\alpha, \beta$-unsaturated $\alpha$-pyrone ring in coumarin derivatives. Thus, the reaction of (I) with phosphoryl chloride in DMF afforded 4-chloro-3-formyl-(2-H)-1-benzopyran2-one derivatives $\left(\mathbf{X I I}_{\mathrm{a}-\mathrm{c}}\right)$. The hydroxyl group at positon-4-in the $\alpha$-pyrone ring seemed to be a good leaving group towards the halogen nucleophile and the behavior of the $\alpha$-pyrone ring as a nucleophile was established by the reaction with DMF afforded the correspondtng 4-chloro-3-formyl coumarin ( $\left.\mathbf{X I I}_{\mathbf{a}-\mathrm{c}}\right)$. It has been reported $^{(14)}$ that ammonia causes ring opening of the $\alpha$-pyrone moiety in coumarin.

Similarly, the reaction of 4-azidocoumarins with nitrogen nucleophiles affected $\alpha$-pyrone ring fission ${ }^{(15)}$. Thus, the reaction of 4-chloro-3-formyl coumarin $\left(\mathbf{X I I}_{\mathbf{a}-\mathrm{c}}\right)$ with sodium azide in DMF afforded the corresponding 4-azido-3-formyl-1benzopyran-2-one (XIII).

The reaction of XIII with active methylene compounds such as, malononitrile and/or ethylcyanoacetate in boiling ethanol containing a few drops of piperidine afforded nitrogen bridge head derivatives, which were defined as 3-cyano-6-oxo-[1] benzopyrano[4,3-d] pyrimidino [3,2-c] triazole $\left(\mathbf{X V I}_{\mathbf{a}-\mathrm{d}}\right)$

The reaction was believed to proceed via the formation of 3-formyl-4aminotriazolyl coumarin intermediate which underwent intramolecular cyclo condensation gave the desired products.

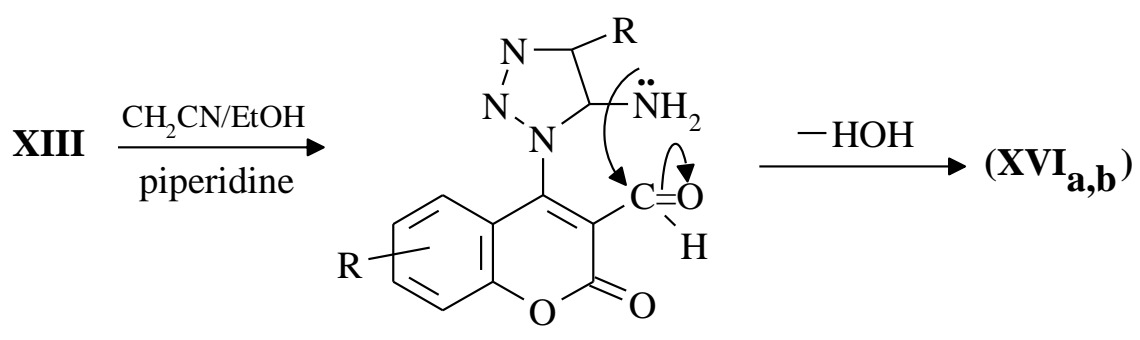

$\mathrm{a}, \mathrm{R}=\mathrm{CN} \quad, \mathrm{b}, \mathrm{R}=\mathrm{COOEt}$

Hydrazinolysis of compound (XII) with hydrazine hydrate in boiling ethanol afforded 1-aryl-4-oxo-[1] benzopyrano[4,3-c] pyrazole $\left(\mathbf{X I V}_{\mathbf{a - d}}\right)$. 
On the other hand the reaction of hydroxylamine hydrochloride with (XII) in boiling ethanol containing few drops of piperidine yielded the corresponding oxime via a nucleophilic attack at the position-3 $\left(\mathbf{X V}_{\mathbf{a}, \mathbf{b}}\right)$.

\section{Experimental}

All melting points were measured in capillary tubes on a GK apparatus and are uncorrected. The IR spectra were recorded in $\mathrm{KBr}$ disks (Wafer technique) on a Shimadzu IR-740 spectrophotometer. The ${ }^{1} \mathrm{H}-\mathrm{NMR}$ spectra were measured on a Perkin-Elmer 360L $60 \mathrm{MHz}$ spectrometer with DMSO- $\mathrm{d}_{6}$ as a solvent, TMS as internal standard and chemical shifts expressed in $\delta \mathrm{ppm}$. The mass spectra were measured on a Finnigan GC/MS INCOS XL spectrometer at 70eV.

\section{Synthesis of 3- Acetyl-4-hydroxy coumarin ( $\left.\mathrm{II}_{\mathrm{a}-\mathrm{c}}\right)$.}

They were prepared according to a previous publication ${ }^{(16,17)}$, c.f. Table 1.

\section{Synthesis of 4-Hydroxy-3-(coumarin-3-yl) pyrazoles $\left(\mathrm{IV}_{\mathrm{a}-\mathrm{f}}\right)$}

To a solution of $\mathbf{I I}_{\mathrm{a}-\mathrm{c}}(0.01 \mathrm{~mol})$ in $15 \mathrm{ml}$ of DMF hydrazine hydrate, phenyl hydrazine and/or 4-nitrophenylhydrazine $(0.01 \mathrm{~mol})$ in $10 \mathrm{ml}$ of absolute ethanol containing a few drops of piperidine was added. The reaction mixture was kept at room temperature overnight, then filtered off, washed with ethanol and dried to give the hydrazone ( III $\left._{\mathbf{a}-\mathrm{f}}\right)$. Compounds ( $\mathbf{I I I}_{\mathrm{a}-\mathrm{f}}$ ) each was taken in $15 \mathrm{ml}$ of DMF and 15 $\mathrm{ml}$ of ethanol containing few drops of piperidine was heated under reflux for 6 hours, then left to cool. The product was collected and recrystallised from the ethanol as $\mathbf{I} \mathbf{V}_{\text {a-f }}$, c.f. Table 1.

\section{Synthesis of 4-Hydroxy-3-(coumarin-3-yl) isoxazol $\left(\mathrm{VI}_{\mathrm{a}, \mathrm{c}}\right),\left(\mathrm{VII}_{\mathrm{a}, \mathrm{b}}\right)$}

A mixture of $\mathbf{I I}_{\mathbf{a}-\mathbf{c}}(0.01 \mathrm{~mol})$ and hydroxylamine hydrochloride $(0.01 \mathrm{~mol})$ in 30 $\mathrm{ml}$ of absolute ethanol containing anhydrous sodium acetate $(0.01 \mathrm{~mol}, 0.82 \mathrm{~g})$ was refluxed for 6 hours, then filtered while hot and left to cool. The product that obtained was collected, washed well with water and recrystalized from the proper solvent to give $\left(\mathbf{V}_{\text {a-c }}\right)$. A mixture of $\left(\mathbf{V}_{\text {a-c }}\right)(0.01 \mathrm{~mol})$ in $20 \mathrm{ml}$ of DMF and $10 \mathrm{ml}$ of ethanol containing few drops of piperidine was heated under reflux for 6 hours, then left to cool. The products VIa-c were separated by filtration recrystallised from the proper solvent in a yield 45-60\%, while the filtrate contained VIIa,b recrystallised from the proper solvent recrystallised from the proper solvent in yield 20-25\% c.f. Table 1. 


\section{Synthesis of 3- Cinnamoyl-4-Hydroxycoumarin (VIII $\left.{ }_{\mathrm{a}-\mathrm{k}}\right)$}

To a solution $\mathbf{I I}_{\mathbf{a}-\mathbf{c}}(0.01 \mathrm{~mol})$ in $30 \mathrm{ml}$ of absolute ethanol containing few drops of piperidine add a solution of the aromatic aldehyde namely, 4-nitrobenzaldehyde, 4-dimethylamineobenzaldehdye, 2-furaldehyde, isonicotinaldehyde, 4chlorobenzaldehyde, 4-methoxy benzaldehyde, and/or 4-methylbenzaldehyde (0.01 mol) in $10 \mathrm{ml}$ of absolute ethanol while stirring. Stirring was continued for 2 hours and the solution was rendered alkaline to litmus, then left to cool overnight. It was then acidified with dil. $\mathrm{Hcl}$ and the precipitate was collected, washed well with light petrol, dried and recrystallised from the proper solvents $\mathbf{V I I I}_{\mathbf{a - k}} \mathbf{c . f}$. Table 1 .

Synthesis of 3- (1-Substituted)-5'-arylpyrazo-3'yl-4-hydroxycoumarin (IX a-h $_{\text {-h }}$ ).

To a solution of VIII $_{\text {a-k }}(0.01 \mathrm{~mol})$ in $50 \mathrm{ml}$ of ethanol-butanol (1:1 involume), add hydrazines namely hydrazine hydrate and/or phenyl hydrazine $(0.01 \mathrm{~mol})$. The mixture was heated under reflux for 6 hours, then cooled and the product was collected and washed well with light petrol and recrystallized from the proper solvent as $\left(\mathbf{I X}_{\mathbf{a}-\mathrm{h}}\right)$ c.f. Table 1.

\section{Synthesis of 3-(1'-acetyl)-5'-arylpyrazol-3'-yl-4-hydrazol-3'yl-4-} hydroxycoumarin $\left(\mathbf{I X} \mathrm{i}_{, \mathbf{j}}\right)$.

To a solution of VIII $_{\mathbf{a}}, \mathbf{V I I I}_{\mathbf{c}},(0.01 \mathrm{~mol})$ in 25 of glacial acetic acid add hydrazine hydrate $(0.01 \mathrm{~mol})$ and the reaction mixture was heated under reflux for 6 hours, then cooled and the product was collected washed with little ethanol, dried and recrystallized from the proper solvent as $\mathbf{I X i}, \mathbf{j}$; c.f. Table 1.

Synthesis of 6-(4'-hydroxy substituted coumarin-3'yl)-1,2-dihydro-2-oxo-4-aryl nicotinonitrile $\left(\mathbf{X I}_{\mathrm{a}-\mathrm{f}}\right)$.

To a solution of VIII $_{\text {a-f }}(0.01 \mathrm{~mol})$ in $20 \mathrm{ml}$ of ethanol add a solution of malononitrile $(0.01 \mathrm{~mol})$ in $20 \mathrm{~mol}$ of ethanol and ammounium acetate $(0.04 \mathrm{~mol})$ and the mixture was heated under reflux for 4 hours. After cooling, the product was poured into $\mathrm{H}_{2} \mathrm{O}(50 \mathrm{ml})$, stirred well and the solid obtained was collected, dried and recrystallized from the proper solvent as $\mathbf{X I}_{\mathbf{a}-\mathbf{f}}$; c.f. Table 1 .

Reaction of VIII with hydroxyl amine hydrochloride. Formation of 3(isoxazole-5') yl coumarine $\left(X_{\mathrm{a}-\mathrm{c}}\right)$.

To a solution of $(0.01 \mathrm{~mol}$ of VIII $\mathbf{a , g}, \mathbf{j})$ in $50 \mathrm{ml}$ pyridine, add $0.01 \mathrm{~mol}$ of hydroxyl amine hydrochloride. the mixture was Refluxed for $3 \mathrm{hrs}$. Cool the mixture 
, then add cold dil Hcl. The solid obtained was collected, dried and recrystallzied from the proper solvent as (Xa-c); c.f. table 1.

Reaction of $\left(I_{a-c}\right)$ with phosphoryl chloride . Formation of 4-chloro-3-formyl coumarin $\left(\mathbf{X I I}_{\mathrm{a}-\mathrm{c}}\right)$.

To 0.01 , mole of $\mathbf{I}$ in $50 \mathrm{ml}$ DMF added $5 \mathrm{ml}$ of phosphoryl chloride then refluxed the mixture for 3 hours on water bath. Cool the mixture and add 1:1 mixture of ice a conc. Hcl. The solid obtained was collected, dried and crystallized from the proper solvent as $\left(\mathbf{X I I}_{\mathrm{a}-\mathrm{c}}\right)$; c.f. table (1).

Reaction of 4-chloro-3-formyl coumarine $\left(\mathrm{XII}_{\mathrm{a}-\mathrm{c}}\right)$ with sodium azide. Formation of 4-azido-3-formyl-2H-1benzopyran-2-one (XIII ${ }_{\mathrm{a}-\mathrm{c}}$ ).

To (0.01 mole) of XII in $50 \mathrm{ml}$ of DMF add ( 0.01 mole) of sod azide. the mixture was refluxed for $3 \mathrm{hrs}$. The solid obtained was collected after cooling dried and recrysallized from the proper solvent as $\left(\mathbf{X I I}_{\mathbf{a}-\mathrm{c}}\right)$; c.f. table 1 .

Reaction of XIII with active methylene compounds . Formation of 3-cyano-6oxo [1] benzopyrano [4,3-d] pyrimidino [3,2-c] triazole $\left(\mathrm{XVI}_{\mathrm{a}-\mathrm{d}}\right)$.

To (0.01 mole) of XIII in $50 \mathrm{ml} \mathrm{EtOH}$ added ( $0.01 \mathrm{~mole})$ of active methylene compounds such as, malononitrile and/ or ethylcyanoacetate in the presence of a few drops of piperidine. the mixture was refluxed for 6 hours. The solid obtained was collected after cooling and recrystallized from the proper solvent as $\left(\mathbf{X V I}_{\mathbf{a}-\mathbf{d}}\right)$; c.f. table (1).

Reaction of 4-chloro-3-formyl coumarin XII with hydrazine hydrate. Formation of 1-aryl-4-oxo-[1] benzopyrano [4,3-c] pyrazole (XIV $\left.\mathrm{X}_{\mathrm{a}-\mathrm{d}}\right)$

To (0.01 mol) of XII in $50 \mathrm{ml}$ dioxan add (0.01 mole) of hydrazines namely hydrazine hydrate, phenyl hydrazine and/or 4-nitrophenylhydrazine. the mixture was Refluxed for 6 hours. The solid obtained was collected after cooling and recrystallized from the proper solvent as $\left(\mathbf{X I V}_{\mathbf{a - d}}\right)$ c.f. table (1).

Reaction of 4-chloro-3-formyl coumarin XII with hydroxyl amine. Formation of 4-chloro-3-oximo coumarin $X \mathrm{~V}_{\mathrm{a}, \mathrm{b}}$.

To (0.01 mole) of XII in $50 \mathrm{ml}$ ethanol in presence of sodium ethoxide add (0.01 mol) of hydroxylamine hydrochloride. the mixture was Refluxed for 6 hours. The solid obtained was collected and recrystallized from the proper solvent as $\mathbf{X V}_{\mathbf{a}, \mathbf{b}} ;$ c.f. table (1). 
Table I. Physical data of the prepared compounds

\begin{tabular}{|c|c|c|c|c|c|c|}
\hline \multirow{2}{*}{$\begin{array}{l}\text { Compd } \\
\text { No. }\end{array}$} & \multirow{2}{*}{$\begin{array}{l}\text { M.P. }{ }^{\circ} \mathrm{C} \\
\text { solvent of } \\
\text { crystallizati } \\
\text { on }\end{array}$} & \multirow{2}{*}{$\begin{array}{l}\text { Molecular } \\
\text { formula } \\
\text { (Mol. wt.) }\end{array}$} & \multicolumn{4}{|c|}{ "Analysis calcd./Found \% } \\
\hline & & & $\mathrm{C}$ & $\mathrm{H}$ & $\mathrm{N}$ & $\mathrm{X}$ \\
\hline $\mathbf{I}_{\mathbf{a}}$ & $\begin{array}{l}101 \\
\mathrm{E}\end{array}$ & $\begin{array}{l}\mathrm{C}_{10} \mathrm{H}_{8} \mathrm{O}_{3} \\
(176)\end{array}$ & $\begin{array}{l}68.19 \\
68.45\end{array}$ & $\begin{array}{l}4.55 \\
4.57\end{array}$ & $\begin{array}{l}-- \\
--\end{array}$ & $\begin{array}{l}-- \\
--\end{array}$ \\
\hline $\mathbf{I}_{\mathbf{b}}$ & $\begin{array}{l}112 \\
\mathrm{E}\end{array}$ & $\begin{array}{l}\mathrm{C}_{10} \mathrm{H}_{8} \mathrm{O}_{4} \\
(192)\end{array}$ & $\begin{array}{l}62.50 \\
62.72\end{array}$ & $\begin{array}{l}4.17 \\
4.25\end{array}$ & $\begin{array}{l}- \\
--\end{array}$ & \\
\hline$\overline{\mathbf{I}_{\mathbf{c}}}$ & $\begin{array}{l}132 \\
\mathrm{E}\end{array}$ & $\begin{array}{l}\mathrm{C}_{9} \mathrm{H}_{5} \mathrm{O}_{3} \mathrm{Br} \\
(240)\end{array}$ & $\begin{array}{l}45.00 \\
45.23\end{array}$ & $\begin{array}{l}2.08 \\
2.22\end{array}$ & $\begin{array}{l}-- \\
--\end{array}$ & $\begin{array}{l}32.92 \\
33 . .19\end{array}$ \\
\hline $\mathbf{I I}_{\mathbf{a}}$ & $\begin{array}{l}152 \\
\mathrm{E}\end{array}$ & $\begin{array}{l}\mathrm{C}_{12} \mathrm{H}_{10} \mathrm{O}_{4} \\
(218)\end{array}$ & $\begin{array}{l}66.06 \\
66.24\end{array}$ & $\begin{array}{l}4.59 \\
4.75\end{array}$ & $\begin{array}{l}- \\
--\end{array}$ & $\begin{array}{l}- \\
--\end{array}$ \\
\hline $\mathbf{I I}_{\mathrm{b}}$ & $\begin{array}{l}161 \\
E\end{array}$ & $\begin{array}{l}\mathrm{C}_{12} \mathrm{H}_{10} \mathrm{O}_{5} \\
(234)\end{array}$ & $\begin{array}{l}61.54 \\
61.75\end{array}$ & $\begin{array}{l}4.27 \\
4.35\end{array}$ & $\begin{array}{l}-- \\
--\end{array}$ & \\
\hline $\mathbf{I I}_{\mathbf{c}}$ & $\begin{array}{l}168 \\
E\end{array}$ & $\begin{array}{l}\mathrm{C}_{11} \mathrm{H}_{7} \mathrm{O}_{4} \mathrm{Br} \\
(274)\end{array}$ & $\begin{array}{l}48.18 \\
48.44\end{array}$ & $\begin{array}{l}2.55 \\
2.60\end{array}$ & $\begin{array}{l}- \\
--\end{array}$ & $\begin{array}{l}28.83 \\
28.95\end{array}$ \\
\hline III $_{\mathrm{a}}$ & $\begin{array}{l}138 \\
\text { P.E. }\end{array}$ & $\begin{array}{l}\mathrm{C}_{18} \mathrm{H}_{16} \mathrm{~N}_{2} \mathrm{O}_{3} \\
(308)\end{array}$ & $\begin{array}{l}70.13 \\
70.35\end{array}$ & $\begin{array}{l}5.19 \\
5.35\end{array}$ & $\begin{array}{l}9.09 \\
9.33\end{array}$ & $\begin{array}{l}- \\
--\end{array}$ \\
\hline III $_{b}$ & $\begin{array}{l}149 \\
\text { P.F. }\end{array}$ & $\begin{array}{l}\mathrm{C}_{18} \mathrm{H}_{15} \mathrm{~N}_{3} \mathrm{O}_{5} \\
(353)\end{array}$ & $\begin{array}{l}61.19 \\
61.20\end{array}$ & $\begin{array}{l}4.25 \\
4.12\end{array}$ & $\begin{array}{l}11.89 \\
11.98\end{array}$ & $\begin{array}{l}-- \\
--\end{array}$ \\
\hline III $_{\mathrm{c}}$ & $\begin{array}{l}115 \\
\text { P.E. }\end{array}$ & $\begin{array}{l}\mathrm{C}_{18} \mathrm{H}_{14} \mathrm{~N}_{4} \mathrm{O}_{7} \\
(398)\end{array}$ & $\begin{array}{l}54.27 \\
54.45\end{array}$ & $\begin{array}{l}3.52 \\
3.50\end{array}$ & $\begin{array}{l}14.07 \\
14.25\end{array}$ & $\begin{array}{l}-- \\
--\end{array}$ \\
\hline III $_{d}$ & $\begin{array}{l}162 \\
E\end{array}$ & $\begin{array}{l}\mathrm{C}_{12} \mathrm{H}_{12} \mathrm{~N}_{2} \mathrm{O}_{4} \\
(248)\end{array}$ & $\begin{array}{l}58.06 \\
58.28\end{array}$ & $\begin{array}{l}4.83 \\
4.95\end{array}$ & $\begin{array}{l}11.29 \\
11.43\end{array}$ & $\begin{array}{l}-- \\
--\end{array}$ \\
\hline III $_{e}$ & $\begin{array}{l}157 \\
\mathrm{E}\end{array}$ & $\begin{array}{l}\mathrm{C}_{18} \mathrm{H}_{16} \mathrm{~N}_{2} \mathrm{O}_{4} \\
\text { (324) }\end{array}$ & $\begin{array}{l}66.67 \\
66.75\end{array}$ & $\begin{array}{l}4.94 \\
4.73 \\
\end{array}$ & $\begin{array}{l}8.64 \\
8.60\end{array}$ & \\
\hline IIII $_{\mathrm{f}}$ & $\begin{array}{l}168 \\
\mathrm{E}\end{array}$ & $\begin{array}{l}\mathrm{C}_{18} \mathrm{H}_{14} \mathrm{~N}_{4} \mathrm{O}_{8} \\
(414)\end{array}$ & $\begin{array}{l}52.17 \\
52.36\end{array}$ & $\begin{array}{l}3.38 \\
3.42\end{array}$ & $\begin{array}{l}13.53 \\
13.62\end{array}$ & \\
\hline III $_{g}$ & $\begin{array}{l}111 \\
\text { P.E. }\end{array}$ & $\begin{array}{l}\mathrm{C}_{11} \mathrm{H}_{9} \mathrm{~N}_{2} \mathrm{O}_{3} \mathrm{Br} \\
(296)\end{array}$ & $\begin{array}{l}44.59 \\
44.68\end{array}$ & $\begin{array}{l}3.04 \\
3.20\end{array}$ & $\begin{array}{l}9.46 \\
9.50\end{array}$ & $\begin{array}{l}26.69 \\
26.70\end{array}$ \\
\hline III $_{h}$ & $\begin{array}{l}139 \\
\mathrm{E}\end{array}$ & $\begin{array}{l}\mathrm{C}_{17} \mathrm{H}_{13} \mathrm{~N}_{2} \mathrm{O}_{3} \mathrm{Br} \\
(372)\end{array}$ & $\begin{array}{l}54.84 \\
55.13\end{array}$ & $\begin{array}{l}3.49 \\
3.30\end{array}$ & $\begin{array}{l}7.53 \\
7.57\end{array}$ & $\begin{array}{l}21.24 \\
21.20\end{array}$ \\
\hline $\mathbf{I V}_{\mathbf{a}}$ & $\begin{array}{l}163 \\
E\end{array}$ & $\begin{array}{l}\mathrm{C}_{19} \mathrm{H}_{14} \mathrm{~N}_{2} \mathrm{O} \\
(318)\end{array}$ & $\begin{array}{l}71.70 \\
71.88\end{array}$ & $\begin{array}{l}4.40 \\
4.42\end{array}$ & $\begin{array}{l}8.81 \\
8.79\end{array}$ & $\begin{array}{l}-- \\
--\end{array}$ \\
\hline$I V_{b}$ & $\begin{array}{l}172 \\
\mathrm{E}\end{array}$ & $\begin{array}{l}\mathrm{C}_{19} \mathrm{H}_{13} \mathrm{~N}_{3} \mathrm{O}_{5} \\
(363)\end{array}$ & $\begin{array}{l}62.81 \\
62.89\end{array}$ & $\begin{array}{l}3.58 \\
3.60\end{array}$ & $\begin{array}{l}11.57 \\
11.60\end{array}$ & $\begin{array}{l}- \\
--\end{array}$ \\
\hline $\mathbf{I V}_{\mathbf{c}}$ & $\begin{array}{l}159 \\
\mathrm{E}\end{array}$ & $\begin{array}{l}\mathrm{C}_{13} \mathrm{H}_{10} \mathrm{~N}_{2} \mathrm{O}_{4} \\
(258)\end{array}$ & $\begin{array}{l}60.47 \\
60.65 \\
\end{array}$ & $\begin{array}{l}3.88 \\
3.67\end{array}$ & $\begin{array}{l}10.85 \\
10.90\end{array}$ & $\begin{array}{l}-- \\
--\end{array}$ \\
\hline $\mathbf{I V}_{\mathrm{d}}$ & $\begin{array}{l}168 \\
\mathrm{E}\end{array}$ & $\begin{array}{l}\mathrm{C}_{19} \mathrm{H}_{14} \mathrm{~N}_{2} \mathrm{O}_{4} \\
(334)\end{array}$ & $\begin{array}{l}68.26 \\
68.34\end{array}$ & $\begin{array}{l}4.20 \\
4.21\end{array}$ & $\begin{array}{l}8.40 \\
8.38\end{array}$ & $\begin{array}{l}-- \\
--\end{array}$ \\
\hline $\mathbf{I V}_{\mathrm{e}}$ & $\begin{array}{l}131 \\
\mathrm{E}\end{array}$ & $\begin{array}{l}\mathrm{C}_{12} \mathrm{H}_{7} \mathrm{~N}_{2} \mathrm{O}_{3} \mathrm{Br} \\
(306)\end{array}$ & $\begin{array}{l}47.06 \\
47.31\end{array}$ & $\begin{array}{l}2.29 \\
2.38\end{array}$ & $\begin{array}{l}9.15 \\
9.13\end{array}$ & $\begin{array}{l}25.81 \\
25.82\end{array}$ \\
\hline $\mathbf{I V}_{\mathrm{f}}$ & $\begin{array}{l}188 \\
\mathrm{E}\end{array}$ & $\begin{array}{l}\mathrm{C}_{18} \mathrm{H}_{11} \mathrm{~N}_{2} \mathrm{O}_{3} \mathrm{Br} \\
(382)\end{array}$ & $\begin{array}{l}56.54 \\
56.62\end{array}$ & $\begin{array}{l}2.88 \\
2.95\end{array}$ & $\begin{array}{l}7.33 \\
7.32\end{array}$ & $\begin{array}{l}20.68 \\
20.70\end{array}$ \\
\hline $\mathbf{V I}_{\mathbf{a}}$ & $\begin{array}{l}128 \\
\text { P.E. }\end{array}$ & $\begin{array}{l}\mathrm{C}_{13} \mathrm{H}_{9} \mathrm{NO}_{4} \\
(243)\end{array}$ & $\begin{array}{l}64.20 \\
64.38\end{array}$ & $\begin{array}{l}3.70 \\
3.68\end{array}$ & $\begin{array}{l}5.76 \\
5.73\end{array}$ & $\begin{array}{l}- \\
--\end{array}$ \\
\hline $\mathbf{V I}_{\mathrm{b}}$ & $\begin{array}{l}159 \\
\mathrm{E}\end{array}$ & $\begin{array}{l}\mathrm{C}_{13} \mathrm{H}_{9} \mathrm{NO}_{5} \\
(259)\end{array}$ & $\begin{array}{l}60.23 \\
60.40 \\
\end{array}$ & $\begin{array}{l}3.50 \\
3.37 \\
\end{array}$ & $\begin{array}{l}5.41 \\
5.40 \\
\end{array}$ & $\begin{array}{l}-- \\
--\end{array}$ \\
\hline $\mathbf{V I}_{\mathbf{c}}$ & $\begin{array}{l}83 \\
\text { P.E. }\end{array}$ & $\begin{array}{l}\mathrm{C}_{12} \mathrm{H}_{6} \mathrm{NO}_{4} \mathrm{Br} \\
(307)\end{array}$ & $\begin{array}{l}46.91 \\
47.06\end{array}$ & $\begin{array}{l}2.93 \\
2.90\end{array}$ & $\begin{array}{l}4.56 \\
4.58\end{array}$ & $\begin{array}{l}25.73 \\
25.70\end{array}$ \\
\hline VII $_{a_{2}}$ & $\begin{array}{l}67 \\
\text { P.E. }\end{array}$ & $\begin{array}{l}\mathrm{C}_{12} \mathrm{H}_{9} \mathrm{NO}_{3} \\
(215)\end{array}$ & $\begin{array}{l}66.98 \\
67.17\end{array}$ & $\begin{array}{l}4.19 \\
4.37\end{array}$ & $\begin{array}{l}6.51 \\
6.50\end{array}$ & -- \\
\hline $\mathrm{VII}_{\mathrm{b}}$ & $\begin{array}{l}101 \\
\text { P.E. }\end{array}$ & $\begin{array}{l}\mathrm{C}_{12} \mathrm{H}_{9} \mathrm{NO}_{4} \\
(231)\end{array}$ & $\begin{array}{l}62.34 \\
62.56 \\
\end{array}$ & $\begin{array}{l}3.90 \\
4.18 \\
\end{array}$ & $\begin{array}{l}6.06 \\
6.10 \\
\end{array}$ & $\begin{array}{l}- \\
--\end{array}$ \\
\hline $\mathbf{I X}_{\mathbf{a}}$ & $\begin{array}{l}154 \\
\mathrm{E}\end{array}$ & $\begin{array}{l}\mathrm{C}_{19} \mathrm{H}_{13} \mathrm{~N}_{3} \mathrm{O}_{5} \\
(363)\end{array}$ & $\begin{array}{l}62.81 \\
63.18\end{array}$ & $\begin{array}{l}3.58 \\
3.60\end{array}$ & $\begin{array}{l}11.57 \\
11.54\end{array}$ & \\
\hline $\mathbf{I X}_{\mathbf{b}}$ & $\begin{array}{l}162 \\
\mathrm{E}\end{array}$ & $\begin{array}{l}\mathrm{C}_{25} \mathrm{H}_{17} \mathrm{~N}_{3} \mathrm{O}_{5} \\
(439)\end{array}$ & \begin{tabular}{|l|}
68.34 \\
68.53 \\
\end{tabular} & $\begin{array}{l}3.87 \\
3.93 \\
\end{array}$ & $\begin{array}{l}9.57 \\
9.60 \\
\end{array}$ & $\begin{array}{l}- \\
-- \\
\end{array}$ \\
\hline & & $\mathrm{C}_{21} \mathrm{H}_{19} \mathrm{~N}_{3} \mathrm{O}_{3}$ & 69.81 & 5.26 & 11.63 & -- \\
\hline
\end{tabular}




\begin{tabular}{|c|c|c|c|c|c|c|}
\hline \multirow{2}{*}{$\begin{array}{l}\text { Compd } \\
\text { No. }\end{array}$} & \multirow{2}{*}{$\begin{array}{l}\text { M.P. }{ }^{\circ} \mathrm{C} \\
\text { solvent of } \\
\text { crystallizati } \\
\text { on }\end{array}$} & \multirow{2}{*}{$\begin{array}{l}\text { Molecular } \\
\text { formula } \\
\text { (Mol. wt.) }\end{array}$} & \multicolumn{4}{|c|}{ "Analysis calcd./Found \% } \\
\hline & & & $\bar{C}$ & $\mathrm{H}$ & $\mathrm{N}$ & $\mathrm{X}$ \\
\hline $\mathbf{I} \mathbf{X}_{\mathrm{c}}$ & $\begin{array}{l}157 \\
\mathrm{E}\end{array}$ & (361) & 69.98 & 5.33 & 11.65 & -- \\
\hline $\mathbf{I} \mathbf{X}_{\mathbf{d}}$ & $\begin{array}{l}169 \\
\mathrm{E}\end{array}$ & $\begin{array}{l}\mathrm{C}_{29} \mathrm{H}_{23} \mathrm{~N}_{3} \mathrm{O}_{3} \\
(461)\end{array}$ & $\begin{array}{l}75.49 \\
75.60\end{array}$ & $\begin{array}{l}4.50 \\
4.42\end{array}$ & $\begin{array}{l}9.11 \\
9.00\end{array}$ & $\begin{array}{l}- \\
--\end{array}$ \\
\hline $\mathbf{I X} \mathbf{X}_{\mathrm{e}}$ & $\begin{array}{l}170 \\
\mathrm{E}\end{array}$ & $\begin{array}{l}\mathrm{C}_{19} \mathrm{H}_{13} \mathrm{~N}_{3} \mathrm{O}_{6} \\
(379)\end{array}$ & $\begin{array}{l}60.16 \\
60.35\end{array}$ & $\begin{array}{l}3.43 \\
3.40\end{array}$ & $\begin{array}{l}11.08 \\
11.21\end{array}$ & $\begin{array}{l}- \\
--\end{array}$ \\
\hline $\mathbf{I X}_{\mathbf{f}}$ & $\begin{array}{l}162 \\
\mathrm{E}\end{array}$ & $\begin{array}{l}\mathrm{C}_{25} \mathrm{H}_{17} \mathrm{~N}_{3} \mathrm{O}_{6} \\
(455)\end{array}$ & $\begin{array}{l}65.93 \\
66.12\end{array}$ & $\begin{array}{l}3.74 \\
3.70\end{array}$ & $\begin{array}{l}9.23 \\
9.33\end{array}$ & $\begin{array}{l}- \\
--\end{array}$ \\
\hline $\mathbf{I X} \mathbf{X}_{\mathrm{g}}$ & $\begin{array}{l}144 \\
\mathrm{E}\end{array}$ & $\begin{array}{l}\mathrm{C}_{19} \mathrm{H}_{13} \mathrm{~N}_{2} \mathrm{O}_{4} \mathrm{Br} \\
(412)\end{array}$ & $\begin{array}{l}55.34 \\
55.53\end{array}$ & $\begin{array}{l}3.16 \\
3.21\end{array}$ & $\begin{array}{l}6.80 \\
6.99\end{array}$ & $\begin{array}{l}19.17 \\
19.20\end{array}$ \\
\hline $\mathbf{I} \mathbf{X}_{\mathbf{h}}$ & $\begin{array}{l}150 \\
\mathrm{E}\end{array}$ & $\begin{array}{l}\mathrm{C}_{14} \mathrm{H}_{14} \mathrm{~N}_{3} \mathrm{O}_{5} \mathrm{Br} \\
(503)\end{array}$ & $\begin{array}{l}57.26 \\
57.45\end{array}$ & $\begin{array}{l}3.70 \\
3.78\end{array}$ & $\begin{array}{l}8.35 \\
8.34\end{array}$ & $\begin{array}{l}12.71 \\
12.70\end{array}$ \\
\hline $\mathbf{I X}_{\mathbf{i}}$ & $\begin{array}{l}203 \\
\mathrm{E}\end{array}$ & $\begin{array}{l}\mathrm{C}_{21} \mathrm{H}_{13} \mathrm{~N}_{3} \mathrm{O}_{6} \\
(405)\end{array}$ & $\begin{array}{l}62.22 \\
62.49\end{array}$ & $\begin{array}{l}3.70 \\
3.73\end{array}$ & $\begin{array}{l}10.37 \\
10.40\end{array}$ & $\begin{array}{l}- \\
--\end{array}$ \\
\hline $\mathbf{I} \mathbf{X}_{\mathbf{j}}$ & $\begin{array}{l}222 \\
\mathrm{E}\end{array}$ & $\begin{array}{l}\mathrm{C}_{19} \mathrm{H}_{14} \mathrm{~N}_{2} \mathrm{O}_{5} \\
(350)\end{array}$ & $\begin{array}{l}65.14 \\
64.85\end{array}$ & $\begin{array}{l}4.00 \\
4.11\end{array}$ & $\begin{array}{l}8.00 \\
8.11\end{array}$ & $\begin{array}{l}- \\
--\end{array}$ \\
\hline $\mathbf{X}_{\mathbf{a}}$ & $\begin{array}{l}115 \\
\text { P.E. }\end{array}$ & $\begin{array}{l}\mathrm{C}_{17} \mathrm{H}_{13} \mathrm{NO}_{5} \\
(309)\end{array}$ & $\begin{array}{l}66.02 \\
66.21\end{array}$ & $\begin{array}{l}3.56 \\
3.55\end{array}$ & $\begin{array}{l}4.53 \\
4.50\end{array}$ & $\begin{array}{l}- \\
--\end{array}$ \\
\hline $\mathbf{X}_{\mathbf{b}}$ & $\begin{array}{l}124 \\
\text { P.E. }\end{array}$ & $\begin{array}{l}\mathrm{C}_{19} \mathrm{H}_{12} \mathrm{~N}_{2} \mathrm{O}_{7} \\
(380)\end{array}$ & $\begin{array}{l}60.00 \\
60.22\end{array}$ & $\begin{array}{l}3.20 \\
3.15\end{array}$ & $\begin{array}{l}7.37 \\
7.35\end{array}$ & $\begin{array}{l}- \\
--\end{array}$ \\
\hline $\mathbf{X}_{\mathbf{c}}$ & $\begin{array}{l}136 \\
\mathrm{E}\end{array}$ & $\begin{array}{l}\mathrm{C}_{19} \mathrm{H}_{12} \mathrm{NO}_{5} \mathrm{Br} \\
(413)\end{array}$ & $\begin{array}{l}55.21 \\
54.95\end{array}$ & $\begin{array}{l}2.91 \\
2.90\end{array}$ & $\begin{array}{l}3.39 \\
3.40\end{array}$ & $\begin{array}{l}19.13 \\
19.12\end{array}$ \\
\hline $\mathbf{X I}_{\mathbf{a}}$ & $\begin{array}{l}187 \\
\mathrm{E}\end{array}$ & $\begin{array}{l}\mathrm{C}_{22} \mathrm{H}_{13} \mathrm{~N}_{3} \mathrm{O}_{6} \\
(415)\end{array}$ & $\begin{array}{l}63.61 \\
63.78\end{array}$ & $\begin{array}{l}3.13 \\
3.10\end{array}$ & $\begin{array}{l}10.12 \\
10.00\end{array}$ & $\begin{array}{l}- \\
--\end{array}$ \\
\hline $\mathbf{X I}_{\mathbf{b}}$ & $\begin{array}{l}189 \\
\mathrm{E}\end{array}$ & $\begin{array}{l}\mathrm{C}_{24} \mathrm{H}_{18} \mathrm{~N}_{3} \mathrm{O}_{4} \\
(412)\end{array}$ & $\begin{array}{l}69.91 \\
70.4\end{array}$ & $\begin{array}{l}4.37 \\
4.36\end{array}$ & $\begin{array}{l}10.19 \\
10.17\end{array}$ & $\begin{array}{l}- \\
--\end{array}$ \\
\hline $\mathbf{X I}_{\mathbf{c}}$ & $\begin{array}{l}192 \\
\mathrm{E}\end{array}$ & $\begin{array}{l}\mathrm{C}_{22} \mathrm{H}_{13} \mathrm{~N}_{3} \mathrm{O}_{7} \\
(431)\end{array}$ & $\begin{array}{l}61.25 \\
61.17\end{array}$ & $\begin{array}{l}3.02 \\
3.01\end{array}$ & $\begin{array}{l}9.74 \\
9.76\end{array}$ & $\begin{array}{l}- \\
--\end{array}$ \\
\hline $\mathbf{X} \mathbf{I}_{\mathbf{d}}$ & $\begin{array}{l}193 \\
\mathrm{E}\end{array}$ & $\begin{array}{l}\mathrm{C}_{24} \mathrm{H}_{19} \mathrm{~N}_{3} \mathrm{O}_{5} \\
\text { (429) }\end{array}$ & $\begin{array}{l}67.13 \\
67.30\end{array}$ & $\begin{array}{l}4.43 \\
4.42\end{array}$ & $\begin{array}{l}9.79 \\
9.80\end{array}$ & $\begin{array}{l}- \\
--\end{array}$ \\
\hline $\mathbf{X I} \mathbf{I}_{\mathrm{e}}$ & $\begin{array}{l}201 \\
\mathrm{E}\end{array}$ & $\begin{array}{l}\mathrm{C}_{19} \mathrm{H}_{9} \mathrm{~N}_{2} \mathrm{O}_{5} \\
(345)\end{array}$ & $\begin{array}{l}66.17 \\
66.08\end{array}$ & $\begin{array}{l}2.61 \\
2.60\end{array}$ & $\begin{array}{l}8.12 \\
8.10\end{array}$ & $\begin{array}{l}- \\
--\end{array}$ \\
\hline $\mathbf{X I}_{\mathrm{f}}$ & $\begin{array}{l}202 \\
\mathrm{E}\end{array}$ & $\begin{array}{l}\mathrm{C}_{20} \mathrm{H}_{10} \mathrm{~N}_{3} \mathrm{O}_{4} \mathrm{Br} \\
(435)\end{array}$ & $\begin{array}{l}55.17 \\
54.95\end{array}$ & $\begin{array}{l}2.30 \\
2.29\end{array}$ & $\begin{array}{l}9.66 \\
9.70\end{array}$ & $\begin{array}{l}18.16 \\
18.20\end{array}$ \\
\hline $\mathbf{X I I}_{\mathrm{a}}$ & $\begin{array}{l}221 \\
\mathrm{E}\end{array}$ & $\begin{array}{l}\mathrm{C}_{11} \mathrm{H}_{7} \mathrm{O}_{3} \mathrm{Cl} \\
(222.5)\end{array}$ & $\begin{array}{l}59.33 \\
59.52\end{array}$ & $\begin{array}{l}3.15 \\
3.14\end{array}$ & $\begin{array}{l}- \\
--\end{array}$ & $\begin{array}{l}15.96 \\
15.95\end{array}$ \\
\hline $\mathbf{X I I}_{\mathbf{b}}$ & $\begin{array}{l}176 \\
\mathrm{E}\end{array}$ & $\begin{array}{l}\mathrm{C}_{11} \mathrm{H}_{7} \mathrm{O}_{4} \mathrm{Cl} \\
(238.5)\end{array}$ & $\begin{array}{l}55.35 \\
55.54\end{array}$ & $\begin{array}{l}2.94 \\
2.93\end{array}$ & $\begin{array}{l}-- \\
--\end{array}$ & $\begin{array}{l}14.88 \\
14.86\end{array}$ \\
\hline $\mathbf{X I I}_{\mathrm{c}}$ & $\begin{array}{l}174 \\
\mathrm{E}\end{array}$ & $\begin{array}{l}\mathrm{C}_{10} \mathrm{H}_{4} \mathrm{O}_{3} \mathrm{BrCl} \\
(286.5)\end{array}$ & $\begin{array}{l}41.88 \\
42.13\end{array}$ & $\begin{array}{l}1.39 \\
1.40\end{array}$ & $\begin{array}{l}- \\
-\end{array}$ & $\begin{array}{l}27.57 \mathrm{Br} \\
27.60 \mathrm{Br} \\
12.39 \mathrm{Cl}\end{array}$ \\
\hline
\end{tabular}




\begin{tabular}{|c|c|c|c|c|c|c|}
\hline \multirow{2}{*}{$\begin{array}{l}\text { Compd } \\
\text { No. }\end{array}$} & \multirow{2}{*}{$\begin{array}{l}\text { M.P. }{ }^{\circ} \mathrm{C} \\
\text { solvent of } \\
\text { crystallizati } \\
\text { on }\end{array}$} & \multirow{2}{*}{$\begin{array}{l}\text { Molecular } \\
\text { formula } \\
\text { (Mol. wt.) }\end{array}$} & \multicolumn{4}{|c|}{ Analysis calcd./Found \% } \\
\hline & & & $\mathrm{C}$ & $\mathrm{H}$ & $\mathrm{N}$ & $\mathrm{X}$ \\
\hline & & & & & & $12.40 \mathrm{Cl}$ \\
\hline XIII $_{a}$ & $\begin{array}{l}182 \\
\mathrm{E}\end{array}$ & $\begin{array}{l}\mathrm{C}_{11} \mathrm{H}_{7} \mathrm{~N}_{3} \mathrm{O}_{3} \\
(229)\end{array}$ & $\begin{array}{l}57.64 \\
57.88\end{array}$ & $\begin{array}{l}3.06 \\
3.05\end{array}$ & $\begin{array}{l}18.34 \\
18.32\end{array}$ & $\begin{array}{l}- \\
--\end{array}$ \\
\hline $\mathbf{X I I I}_{\mathrm{b}}$ & $\begin{array}{l}179 \\
\mathrm{E}\end{array}$ & $\begin{array}{l}\mathrm{C}_{11} \mathrm{H}_{7} \mathrm{~N}_{3} \mathrm{O}_{4} \\
(245)\end{array}$ & $\begin{array}{l}53.88 \\
54.17\end{array}$ & $\begin{array}{l}2.86 \\
2.85\end{array}$ & $\begin{array}{l}17.14 \\
17.12\end{array}$ & $\begin{array}{l}- \\
--\end{array}$ \\
\hline XIII $_{c}$ & $\begin{array}{l}180 \\
\mathrm{E}\end{array}$ & $\begin{array}{l}\mathrm{C}_{10} \mathrm{H}_{4} \mathrm{~N}_{3} \mathrm{O}_{3} \mathrm{Br} \\
(309)\end{array}$ & $\begin{array}{l}38.83 \\
39.09\end{array}$ & $\begin{array}{l}1.29 \\
1.30\end{array}$ & $\begin{array}{l}13.59 \\
13.60\end{array}$ & $\begin{array}{l}25.57 \\
25.60\end{array}$ \\
\hline $\mathbf{X I V}_{\mathrm{a}}$ & $\begin{array}{l}133 \\
\text { P.E. }\end{array}$ & $\begin{array}{l}\mathrm{C}_{11} \mathrm{H}_{8} \mathrm{O}_{2} \mathrm{~N}_{2} \\
(200)\end{array}$ & $\begin{array}{l}66.00 \\
66.23\end{array}$ & $\begin{array}{l}4.00 \\
4.02\end{array}$ & $\begin{array}{l}14.00 \\
14.03\end{array}$ & $\begin{array}{l}25.00 \\
-- \\
--\end{array}$ \\
\hline $\mathbf{X I V}_{\mathrm{b}}$ & $\begin{array}{l}129 \\
\mathrm{E}\end{array}$ & $\begin{array}{l}\mathrm{C}_{17} \mathrm{H}_{12} \mathrm{~N}_{2} \mathrm{O}_{2} \\
(264)\end{array}$ & $\begin{array}{l}77.27 \\
77.35\end{array}$ & $\begin{array}{l}4.55 \\
4.53\end{array}$ & $\begin{array}{l}10.61 \\
10.63\end{array}$ & $\begin{array}{l}- \\
--\end{array}$ \\
\hline $\mathbf{X I V}_{\mathbf{c}}$ & $\begin{array}{l}130 \\
\mathrm{E}\end{array}$ & $\begin{array}{l}\mathrm{C}_{17} \mathrm{H}_{11} \mathrm{~N}_{3} \mathrm{O}_{4} \\
(321)\end{array}$ & $\begin{array}{l}63.55 \\
63.60\end{array}$ & $\begin{array}{l}3.43 \\
3.42\end{array}$ & $\begin{array}{l}13.08 \\
13.20\end{array}$ & $\begin{array}{l}- \\
--\end{array}$ \\
\hline $\mathbf{X I V}_{\mathrm{d}}$ & $\begin{array}{l}121 \\
\text { P.E. }\end{array}$ & $\begin{array}{l}\mathrm{C}_{17} \mathrm{H}_{12} \mathrm{~N}_{2} \mathrm{O}_{3} \\
(292)\end{array}$ & $\begin{array}{l}69.86 \\
70.09\end{array}$ & $\begin{array}{l}4.11 \\
4.10\end{array}$ & $\begin{array}{l}9.59 \\
9.60\end{array}$ & $\begin{array}{l}-- \\
-- \\
--\end{array}$ \\
\hline $\mathbf{X} \mathbf{V}_{\mathrm{a}}$ & $\begin{array}{l}111 \\
\text { P.E. }\end{array}$ & $\begin{array}{l}\mathrm{C}_{11} \mathrm{H}_{8} \mathrm{NO}_{3} \mathrm{Cl} \\
(237.5)\end{array}$ & $\begin{array}{l}55.58 \\
55.57\end{array}$ & $\begin{array}{l}3.37 \\
3.36\end{array}$ & $\begin{array}{l}5.89 \\
5.90\end{array}$ & $\begin{array}{l}14.95 \\
14.97\end{array}$ \\
\hline $\mathbf{X V _ { b }}$ & $\begin{array}{l}122 \\
\text { P.E. }\end{array}$ & $\begin{array}{l}\mathrm{C}_{11} \mathrm{H}_{8} \mathrm{NO}_{4} \mathrm{Cl} \\
(253.5)\end{array}$ & $\begin{array}{l}52.07 \\
52.22\end{array}$ & $\begin{array}{l}3.20 \\
3.16\end{array}$ & $\begin{array}{l}5.52 \\
5.50\end{array}$ & $\begin{array}{l}14.00 \\
14.03\end{array}$ \\
\hline $\mathbf{X V I}_{\mathrm{a}}$ & $\begin{array}{l}66 \\
\text { P.E. }\end{array}$ & $\begin{array}{l}\mathrm{C}_{14} \mathrm{H}_{7} \mathrm{~N}_{5} \mathrm{O}_{2} \\
(277)\end{array}$ & $\begin{array}{l}60.65 \\
60.84\end{array}$ & $\begin{array}{l}2.53 \\
2.50\end{array}$ & $\begin{array}{l}25.27 \\
25.30\end{array}$ & $\begin{array}{l}-- \\
--\end{array}$ \\
\hline $\mathbf{X V I}_{\mathbf{b}}$ & $\begin{array}{l}89 \\
\text { P.E. }\end{array}$ & $\begin{array}{l}\mathrm{C}_{16} \mathrm{H}_{11} \mathrm{~N}_{4} \mathrm{O}_{4} \\
(323)\end{array}$ & $\begin{array}{l}59.44 \\
59.63\end{array}$ & $\begin{array}{l}3.41 \\
3.40\end{array}$ & $\begin{array}{l}17.34 \\
17.33\end{array}$ & $\begin{array}{l}- \\
--\end{array}$ \\
\hline $\mathbf{X V I}_{\mathrm{c}}$ & $\begin{array}{l}78 \\
\text { P.E. }\end{array}$ & $\begin{array}{l}\mathrm{C}_{14} \mathrm{H}_{7} \mathrm{~N}_{5} \mathrm{O}_{3} \\
(293)\end{array}$ & $\begin{array}{l}57.34 \\
57.38\end{array}$ & $\begin{array}{l}2.39 \\
2.38\end{array}$ & $\begin{array}{l}23.89 \\
23.90\end{array}$ & $\begin{array}{l}- \\
--\end{array}$ \\
\hline $\mathbf{X V I}_{\mathrm{d}}$ & $\begin{array}{l}80 \\
\text { P.E }\end{array}$ & $\begin{array}{l}\mathrm{C}_{16} \mathrm{H}_{11} \mathrm{~N}_{4} \mathrm{O}_{5} \\
(339)\end{array}$ & $\begin{array}{l}56.64 \\
56.43\end{array}$ & $\begin{array}{l}3.24 \\
3.22\end{array}$ & $\begin{array}{l}16.52 \\
16.50\end{array}$ & $\begin{array}{l}- \\
--\end{array}$ \\
\hline
\end{tabular}

\section{Pharmacological activity}

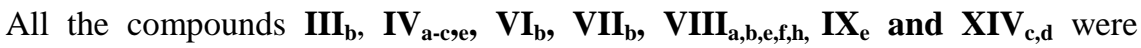
screened for their in vitro antibacterial activity against E.coli and Xanthomonas citri by cup plate method ${ }^{(15,)}$, Summarized in table II. Nutrient agar was melted in a water bath and cooled to $45^{\circ} \mathrm{C}$ with gentle shaking to bring about uniform cooling. It was with $0.5-0.6 \mathrm{ml}$ of culture and mixed well by pouring gentle shaking before onto the sterilized Petri dishes. The poured materials were allowed to set and thereafter the "CUPS" were made by punching into the agar surface with sterile cork borer and scooping out the punched part of the agar. Into these cups were added $0.1 \mathrm{ml}$. portion of the tested compound in the solvent with the help of sterile syringe. The drug solution was allowed to diffuse for about an hour into the medium. The plates were incubated at $37^{\circ} \mathrm{C}$ and the results noted. The standard is (streptomycin) 
Table II. Antibacterial activity data of the tested compounds

\begin{tabular}{|c|c|c|}
\hline $\begin{array}{c}\text { Comp. } \\
\text { No. }\end{array}$ & $\begin{array}{c}\text { E.coli }^{*} \\
\text { (Zone in mm) }\end{array}$ & $\begin{array}{c}\text { X.Citri* }^{*} \\
\text { (zone in } \mathrm{mm} \text { ) }\end{array}$ \\
\hline III $_{\mathrm{b}}$ & 8 & 13 \\
\hline $\mathbf{I V}_{\mathrm{a}}$ & 12 & 15 \\
\hline$I V_{b}$ & 10 & 11 \\
\hline $\mathbf{I V}_{\mathrm{c}}$ & 8 & 10 \\
\hline$I_{\mathrm{e}}$ & 11 & 8 \\
\hline$V I_{b}$ & 9 & 10 \\
\hline VII $_{b}$ & 13 & 12 \\
\hline VIII $_{a}$ & 10 & 13 \\
\hline VIII $_{b}$ & 12 & 14 \\
\hline VIII $_{e}$ & 9 & 9 \\
\hline VIII $_{f}$ & 12 & 8 \\
\hline VIII $_{h}$ & 7 & 13 \\
\hline $\mathbf{I X} \mathbf{X}_{\mathrm{e}}$ & 10 & 11 \\
\hline $\mathbf{X I V}_{\mathbf{c}}$ & 11 & 10 \\
\hline $\mathbf{X I V}_{\mathrm{d}}$ & 12 & 14 \\
\hline
\end{tabular}

* Standard (Streptomycin) 


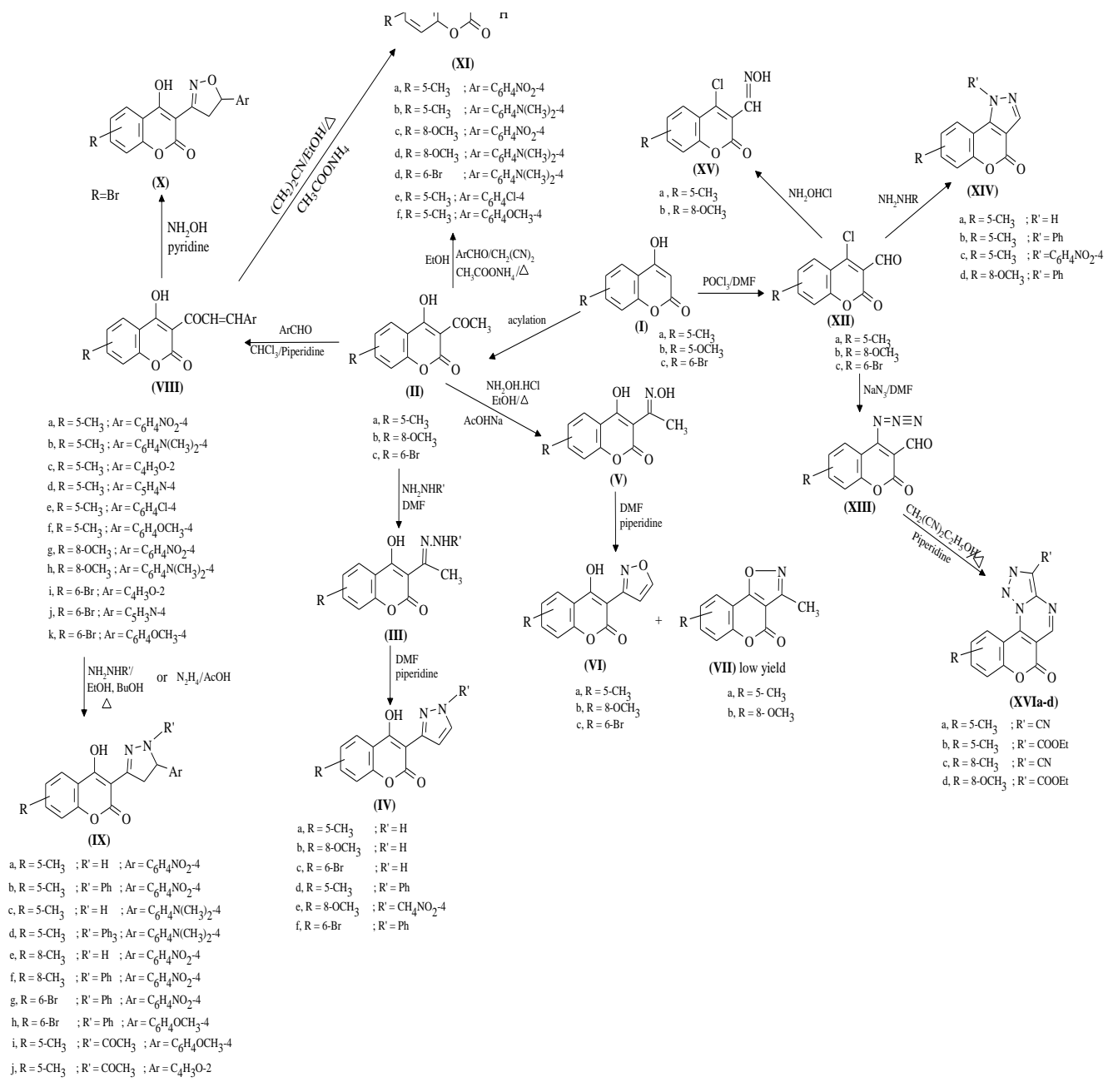




\section{References:}

1. B.RAJITHA, V.NAVEEN KUMAR, P. SOMESHWAR, J. VENUMADHAV, P. NARSHIMA REDDY, AND Y. THIRUPATHI Reddy, Arkivoc 2006 (xii)23-27

2. EL -SAYED, A.M.; GHATTAS, A.B.; EL- WASSIMY, M.T. AND ABD ALLAH, O.A. Ij Farmaco 1999, 54,56.

3. HMCIAR, P.; GAPLOVSKY, A.; AND DONOVALOVA, J. Czech CS 275,270(CL. CO7D 309/38), Appl. 88/5,688; Chem. Abstr. 118,233882j (1993).

4. DARWISH, O.S.; GRANUM, K.A.; TAN, Q. AND HSUNG, R.P. Tetrahedron lett., 42 (2001) 3283.

5. MUSICKI, B.; PERIERS, A.M.; LAURIN, P.; FERROUD, D.; BENEDETTI, Y.; LACHAUD, S.; CHATREAUX, F.; HAESSLEIN, J.L.; NTIS, A.; PIERRE, C.; Khider, J.; TESSOT, N.; AIRAULT, M.; DEMASSEY, J.; DUPUIS-HAMELIN, C.; LASSAINGNE, P.; BONNEFOY, A.; VICAT, P.; AND KLICH, M. Bioorg. Med. Chem. Lett. 2000,10,1695.

6. ITO, C.; ITOIGAWA, M.; KATSUNO, S.; OMURA, 14.; TOKUDA, H.; NISHINO, H.; AND FURUKAWA, H.J. Nat. Prod. 2000,639,1218.

7. KASHMAN, Y .; GUSTAFSON, K.R.; RICHARD, W.; CARDELlINA, J. and Me Mahan, J.B. J. Med. Chem. 1992,35,2735.

8. MEKEE, T.C.; FULLER, R.W.; COVINGTON, C.D.; CARDELLINA, J.H.; GULAKOWSKI, R.J.; KREPPS, B.L.; ME MAHON, J.B.; AND BOYD, M.RJ. Nat. Prod. 1996,59,754.

9. ISHIKAWA, T.; OKU, Y.; TANAKA, T.; AND KUMAMOTO, T.; Tetrahedron lett. $1999,40,3777$.

10. TANAKA, T.; KUMAMOTO, T.; AND ISHIKAWA, T. TETRAHEDRON lett. $2000,41,10229$.

11. ANGElOVA, I.; AND DIMITROVA, E. Org. Prep.Proced. Int. 1989,21(3),341; Chem. Abstr., 6103y(1990).

12. OGANESIAN, E.T.; POGREBNYAK, A.V. AND GRIDNEV, YU.S. Khim. Farm. Zh.1994(11),36; Chem. Abstr.,125,25610m,(1996).

13. ANGEL, A.; LUIS, A.C; ALFONSO, G.O.; CARMEN, S.R.M.; PEDRO, Y.; SANTIAGO, G.G. AND ESTHER, G.R.J. Org. Chem., 1999,(26),9493.

14. O' CALlAGFCAN, C.N.; ME MUNRY, T.B.H. J. Chem. Res., Synop. 1989,(11), 329.

15. Ito, K.; Higuchi, y.; Tame, Ch.; and Hariya, J. Heterocycles 1993, 35(2),937.

16. CHICKSHANK, R.; DUGID, J.P.; MARMON, D.P.; SWAIN, R., H.A. In Medical microbiology, 1975, Vol.2, (Churchill-Livingstone, Edinburgh, London).

17. SAMMOUR, A.; MAREI, A.; AL-ASHNY, S., J. Chem. U.A.R. 13, 281 (1970) 